N. Obata

Nagoya Math. J.

Vol. 129 (1993), 1-22

\title{
WHITE NOISE DELTA FUNCTIONS AND CONTINUOUS VERSION THEOREM
}

\author{
NOBUAKI OBATA
}

\section{Introduction}

The recently developed Hida calculus of white noise [5] is an infinite dimensional analogue of Schwartz' distribution theory besed on the Gelfand triple $(E) \subset\left(L^{2}\right)=L^{2}\left(E^{*}, \mu\right) \subset(E)^{*}$, where $\left(E^{*}, \mu\right)$ is Gaussian space and $\left(L^{2}\right)$ is (a realization of) Fock space. It has been so far discussed aiming at an application to quantum physics, for instance [1], [3], and infinite dimensional harmonic analysis [7], [8], [13], [14], [15]. During the development an important milestone was Kubo-Yokoi's continuous version theorem [11] which asserts that every test white noise functional $\phi \in(E)$ admits a unique continuous version and, therefore, the test functionals constitute a space of continuous functions on $E^{*}$. This theorem is very fundamental and indispensable for many arguments. For example, it allows us to introduce a delta function on Gaussian space, which is one of the most important generalized functions. Furthermore, the continuous version theorem is effectively applied to description of positive generalized white noise functionals [19].

The motivation of this paper is to give an alternative proof of the continuous version theorem by means of a direct use of defining Hilbertian seminorms of $E^{*}$. In fact, this approach yields a sharp estimate of white noise delta functions $\delta_{x} \in$ $(E)^{*}, x \in E^{*}$, from which the continuous version theorem follows. Moreover, with this method we may prove the continuity of $x \mapsto \delta_{x} \in(E)^{*}, x \in E^{*}$, which guarantees that the $n$-fold (topological) tensor product $(E) \otimes \cdots \otimes(E)$ is again a space of continuous functions on the product of the Gaussian space $E^{*} \times \cdots \times$ $E^{*}$ (n times).

Here we remark some closely related works. In [12] Lee proved that each test functional $\phi \in(E)$ admits an analytic version on each Hilbert space $E_{-p}$, where $E^{*}=$ ind $\lim _{p \rightarrow \infty} E_{-p}$. However, since the inductive system $\left\{E_{-p}\right\}_{p \geq 0}$ is not strict, our continuous version theorem does not follow from his result. In [9] Kondrat'ev

Received March 5, 1992. 
and Samoylenko studied smoothness of test functionals on $\mathbf{R}^{\infty}$ equipped with the product Gaussian measure. Although based on a different framework, their discussion can be translated into our language. However, it turns out that their results have little in common with ours but some with Lee's. Finally, within the framework of Malliavin calculus the unique existence of quasi-continuous version has been discussed in many contexts, see e.g., [17] and references cited therein.

The paper is organized as follows. In Section 1 we recapitulate a well known construction of Gelfand triples under the name of standard construction. Section 2 is devoted to a brief review of test and generalized white noise functionals. In Section 3 we formulate the main results. In Section 4 we introduce a set of defining Hilbertian seminorms of $E^{*}$ and in Section 5 we prove the main results. Section 6 contains some results on a tensor product of white noise test functionals.

Notation. If $\mathfrak{X}$ is a real vector space, we denote by $\mathfrak{X}_{\mathbf{C}}$ the complexification. For two vector spaces $\mathfrak{X}$ and $\mathfrak{Y}$ we denote by $\mathfrak{X} \bigotimes_{\text {alg }} \mathfrak{Y}$ their algebraic tensor product. If $\mathfrak{X}=H$ and $\mathfrak{Y}=K$ are Hilbert spaces, we denote by $H \otimes K$ the Hilbert space tensor product. For nuclear spaces $\mathfrak{X}$ and $\mathfrak{Y}$, we denote simply by $\mathfrak{X} \otimes \mathfrak{Y}$ the completion of $\mathfrak{X} \otimes_{\text {alg }} \mathfrak{Y}$ with respect to the $\pi$-topology, i.e., the strongest locally convex topology on $\mathfrak{X} \otimes_{\text {alg }} \mathfrak{Y}$ such that the canonical map $\mathfrak{X} \times \mathfrak{Y} \rightarrow \mathfrak{X} \otimes_{\text {alg }} \mathfrak{Y}$ is continuous. Although the $\pi$-tensor product of Hilbert spaces is different from the Hilbert space tensor product, there will be no confusion. We denote by $\mathfrak{X}^{\hat{\otimes} n} \subset$ $\mathfrak{X}^{\otimes n}$ the closed subspace of symmetric tensor products. We also use $\left(\mathfrak{X}^{\otimes n}\right)_{\text {sym }}^{*}$ for the same meaning in case of the strong dual spaces.

\section{Standard construction of Gelfand triples}

Motivated by the works of Berezansky-Kondrat'ev [2] and Gelfand-Vilenkin [4], we reformulate a useful method of constructing a nuclear Fréchet space or equivalently a Gelfand triple.

Let $H$ be a real separable Hilbert space with norm $|\cdot|_{0}$ and inner product $\langle\cdot, \cdot\rangle$. Assume we are given a pair $\left(\left\{e_{j}\right\}_{j=0}^{\infty},\left\{\lambda_{j}\right\}_{j=0}^{\infty}\right.$ ) of a complete orthonormal basis of $H$ and a sequence of positive numbers with $\sum_{j=0}^{\infty} \lambda_{j}^{-2 r}<\infty$ for some $r>0$. We then put

$$
|\xi|_{p}=\left(\sum_{j=0}^{\infty} \lambda_{j}^{2 p}\left\langle\xi, e_{j}\right\rangle^{2}\right)^{1 / 2}, \quad \xi \in H, p \in \mathbf{R},
$$

though $|\xi|_{p}=\infty$ can happen. For $p \geq 0$ let $E_{p}$ denote the subspace of $\xi \in H$ with $|\xi|_{p}<\infty$. Obviously, $E_{p}$ becomes a Hilbert space with norm $|\cdot|_{p}$. Again for 
$p \geq 0$ let $E_{-p}$ denote the completion of $H$ with respect to the Hilbertian norm $|\cdot|_{-p}$ on $H$. We have thus obtained a chain of Hilbert spaces:

$$
\begin{gathered}
\cdots \subset E_{q} \subset \cdots \subset E_{p} \subset \cdots \subset E_{0}=H \subset \cdots \subset E_{-p} \subset \cdots \subset E_{-q} \subset \cdots, \\
q \geq p \geq 0,
\end{gathered}
$$

where every canonical injection $E_{q} \rightarrow E_{p}, q \geq p$, is continuous and has dense image. As is easily seen, the inner product $\langle\cdot, \cdot\rangle$ of $H$ is naturally extended to the canonical bilinear form on $E_{-p} \times E_{p}, p \geq 0$, through which $E_{-p}$ is identified with the strong dual of $E_{p}$.

TheOREM 1.1. Equipped with the Hilbertian norms $|\cdot|_{p}, p \geq 0, E=\cap_{p \geq 0} E_{p}$ becomes a nuclear Fréchet space, which is isomorphic to the projective limit proj $\lim _{p \rightarrow \infty} E_{p}$. Moreover, the strong dual $E^{*}$ is isomorphic to the inductive limit ind $\lim _{p \rightarrow \infty} E_{-p}$ and is identified with $\cup_{p \geq 0} E_{-p}$ as vector space.

The proof is straightforward, see [4: Chap. I ] and [16: Chap. IV]. We have thus obtained a Gelfand triple $E \subset H \subset E^{*}$ from the pair $\left(\{e,\}_{j=0}^{\infty},\left\{\lambda_{j}\right\}_{j=0}^{\infty}\right)$. This construction will be called standard.

While, it is sometimes more convenient to start with a densely defined operator on $H$ instead of a pair $\left(\left\{e_{j}\right\}_{j=0}^{\infty},\left\{\lambda_{j}\right\}_{j=0}^{\infty}\right)$. A linear operator $A$ with dense domain $\operatorname{Dom}(A) \subset H$ is called standard if there is a complete orthonormal basis $\left\{e_{j}\right\}_{j=0}^{\infty}$ for $H$ contained in $\operatorname{Dom}(A)$ such that

(S1) $A e_{j}=\lambda_{j} e_{j}$ with $\lambda_{j}>0$;

(S2) $\sum_{j=0}^{\infty} \lambda_{j}^{-2 r}<\infty$ for some $r>0$.

The relation between a standard operator $A$ and a pair $\left(\left\{e_{j}\right\}_{j=0}^{\infty},\left\{\lambda_{j}\right\}_{j=0}^{\infty}\right)$ is described as

$$
A \xi=\sum_{j=0}^{\infty} \lambda_{j}\left\langle\xi, e_{j}\right\rangle e_{j}, \quad \xi \in \operatorname{Dom}(A)
$$

Given a standard operator $A$, we construct a Gelfand triple in the standard manner.

Lemma 1.2. If $A$ is a standard operator on $H$, so is $A^{s}$ for any $s>0$ and the resultant Gelfand triples are isomorphic. 
The proof is straightforward. By virtue of Lemma 1.2 we may assume without loss of generality that $r=1$ in (S2), when we discuss standard construction of Gelfand triples.

Let $\Omega$ be a topological space equipped with a Borel measure $\nu$. If $A$ is a standard operator on $H=L^{2}(\Omega, \nu ; \mathbf{R})$, the Gelfand triple constructed from $A$ is explicitly written as

$$
\oiint_{A}(\Omega) \subset L^{2}(\Omega, \nu ; \mathbf{R}) \subset \mathscr{\oiint}_{A}^{*}(\Omega) .
$$

By construction each element of $\mathscr{\bigotimes}_{A}(\Omega)$ is a function on $\Omega$ which is determined up to $\nu$-null functions. For many practical reasons it is desired that $\mathscr{\bigotimes}_{A}(\Omega)$ can be identified with a space of continuous functions on $\Omega$. In this connection we propose the following hypothesis:

(H1) For each function $\xi \in \mathscr{\bigotimes}_{A}(\Omega)$ there exists a unique continuous function $\tilde{\xi}$ on $\Omega$ such that $\xi(\omega)=\tilde{\xi}(\omega)$ for $\nu$-a.e. $\omega \in \Omega$.

Once this condition is satisfied, we always regard $\mathscr{H}_{A}(\Omega)$ as a space of continuous functions on $\Omega$ and we do not use the symbol $\tilde{\xi}$. Under (H1) we consider two more hypotheses:

(H2) For each $\omega \in \Omega$ the evaluation map $\delta_{\omega}: \xi \mapsto \xi(\omega), \xi \in \mathscr{\bigotimes}_{A}(\Omega)$, is a continuous linear functional, i.e., $\delta_{\omega} \in \mathscr{\oiint}_{A}^{*}(\Omega)$.

(H3) The map $\omega \mapsto \delta_{\omega} \in \mathscr{S}_{A}^{*}(\Omega), \omega \in \Omega$, is continuous with respect to the strong dual topology of $\mathscr{S}_{A}^{*}(\Omega)$.

The above hypotheses are motivated by the work of Kubo and Takenaka [10]. While, in [4: Chap. I] the evaluation map $\delta_{\omega}$ is discussed without topological structure of $\Omega$. A sufficient condition for (H1)-(H3) is presented in Appendix.

\section{Generalized white noise functionals}

We keep to the same notation as in $\S 1$. Let $A$ be a standard operator on a real Hilbert space $H$ satisfying

(A1) $A e_{j}=\lambda_{j} e_{j}$ with $\lambda_{j} \in \mathbf{R}$;

(A2) $\sum_{j=0}^{\infty} \lambda_{j}^{-2}<\infty$;

(A3) $1<\lambda_{0} \leq \lambda_{1} \leq \cdots \rightarrow \infty$.

The last condition is indispensable to our white noise calculus setup. Let $E \subset H \subset E^{*}$ be the Gelfand triple constructed from $A$ in the standard manner.

The Gaussian measure $\mu$ on $E^{*}$ is defined by 


$$
\exp \left(-\frac{|\xi|_{0}^{2}}{2}\right)=\int_{E^{*}} e^{i\langle x, \xi\rangle} \mu(d x), \quad \xi \in E
$$

We consider the Hilbert space $L^{2}\left(E^{*}, \mu ; \mathbf{R}\right)$ and its complexification $\left(L^{2}\right)$. Their norms are denoted by $\|\cdot\|_{0}$.

In order to introduce a standard operator on $L^{2}\left(E^{*}, \mu ; \mathbf{R}\right)$ we need a variant of Wiener-Itô decomposition. For $x \in E^{*}$ and $n=0,1,2, \ldots$ we define $: x^{\otimes n}: \in$ $\left(E^{\otimes n}\right)_{\mathrm{sym}}^{*}$ inductively as follows:

$$
\left\{\begin{array}{l}
: x^{\otimes 0}:=1, \\
: x^{\otimes 1}:=x, \\
: x^{\otimes n}:=x \widehat{\otimes}: x^{\otimes(n-1)}:-(n-1) \tau \widehat{\otimes}: x^{\otimes(n-2)}:, \quad n \geq 2,
\end{array}\right.
$$

where $\tau \in(E \otimes E)_{\text {sym }}^{*}$ is the trace uniquely determined by

$$
\langle\tau, \xi \otimes \eta\rangle=\langle\xi, \eta\rangle, \quad \xi, \eta \in E .
$$

Let $f_{n} \in E_{\mathbf{C}}^{\hat{\otimes} n}$. Then $\phi_{n}(x)=\left\langle: x^{\otimes n}:, f_{n}\right\rangle$ becomes a continuous function on $E^{*}$ which satisfies $\left\|\phi_{n}\right\|_{0}^{2}=n !\left|f_{n}\right|_{0}^{2}$. Using this isometry, we may define $\left\langle: x^{\otimes n}:, f_{n}\right\rangle$ for $f_{n} \in H_{\mathbf{C}}^{\hat{\otimes} n}$ in $L^{2}$-sense. With these notations we have the following

Proposition 2.1. For each $\phi \in\left(L^{2}\right)$ there exists a unique sequence $\left(f_{n}\right)_{n=0}^{\infty}, f_{n} \in$ $H_{\mathbf{C}}^{\hat{\otimes} n}$, such that

$$
\phi(x)=\sum_{n=0}^{\infty}\left\langle: x^{\otimes n}:, f_{n}\right\rangle, \quad x \in E^{*},
$$

where the right hand side is an orthogonal direct sum in $\left(L^{2}\right)$. In that case it holds that

$$
\|\phi\|_{0}^{2}=\sum_{n=0}^{\infty} n !\left|f_{n}\right|_{0}^{2}
$$

We now define a second quantized operator $\Gamma(A)$. Let Dom $(\Gamma(A))$ be the space of functions $\phi$ of the form (4) such that $f_{n} \in E^{\widehat{\otimes} n}$ and $f_{n}=0$ except finitely many $n$. For $\phi \in \operatorname{Dom}(\Gamma(A))$ put

$$
\Gamma(A) \phi(x)=\sum_{n=0}^{\infty}\left\langle: x^{\otimes n}:, A^{\otimes n} f_{n}\right\rangle .
$$

Then, $\Gamma(A)$ becomes a standard operator on $L^{2}\left(E^{*}, \mu ; \mathbf{R}\right)$, see [15]. Employing the standard construction, we obtain a new Gelfand triple:

$$
\mathscr{\vartheta}_{\Gamma(A)}\left(E^{*}\right) \subset L^{2}\left(E^{*}, \mu ; \mathbf{R}\right) \subset \mathscr{S}_{\Gamma(A)}^{*}\left(E^{*}\right),
$$


of which complexification is denoted by

$$
(E) \subset\left(L^{2}\right) \subset(E)^{*} .
$$

Elements $\phi \in(E)$ and $\Phi \in(E)^{*}$ are called a test (white noise) functional and a generalized (white noise) functional, respectively. The canonical bilinear form on $(E)^{*} \times(E)$ is denoted by $\langle\cdot \cdot, \cdot\rangle$.

It follows from (5), (6) and the definition of norms that

$$
\|\phi\|_{p}^{2}=\left\|\Gamma(A)^{p} \phi\right\|_{0}^{2}=\sum_{n=0}^{\infty} n !\left|\left(A^{\otimes n}\right)^{p} f_{n}\right|_{0}^{2}=\sum_{n=0}^{\infty} n !\left|f_{n}\right|_{p}^{2} .
$$

We then obtain

Proposition 2.2. Let $\phi \in\left(L^{2}\right)$ be expressed as in (4). Then, $\phi \in(E)$ if and only if $f_{n} \in E_{\mathbf{C}}^{\hat{\otimes} n}$ for all $n=0,1,2, \ldots$ and $\sum_{n=0}^{\infty} n !\left|f_{n}\right|_{p}^{2}<\infty$ for all $p \geq 0$.

\section{Continuous version theorem}

In this section we formulate the main assertions. Recall that by construction each $\phi \in(E)$ is determined only up to $\mu$-null functions.

THEOREM 3.1. For each $\phi \in(E)$ there exists a unique continuous function $\tilde{\phi}$ on $E^{*}$ such that $\phi(x)=\tilde{\phi}(x)$ for $\mu$-a.e. $x \in E^{*}$. Moreover, $\tilde{\phi}(x)$ is given by the absolutely convergent series:

$$
\tilde{\phi}(x)=\sum_{n=0}^{\infty}\left\langle: x^{\otimes n}:, f_{n}\right\rangle, \quad x \in E^{*},
$$

where $\left(f_{n}\right)_{n=0}^{\infty}$ corresponds to the given $\phi$ as in Proposition 2.1 .

The above assertion should be carefully compared with Proposition 2.2 which asserts that the series converges with respect to the norms $\|\cdot\|_{p}, p \geq 0$. By virtue of Theorem 3.1 we always understand $(E)$ to be the space of continuous functions $\phi$ on $E^{*}$ of the form:

$$
\phi(x)=\sum_{n=0}^{\infty}\left\langle: x^{\otimes n}:, f_{n}\right\rangle
$$

where

(i ) $f_{n} \in E_{\mathbf{C}}^{\hat{\otimes} n}$ for $n=0,1,2, \ldots$; 
(ii) $\sum_{n=0}^{\infty} n !\left|f_{n}\right|_{p}^{2}<\infty$ for all $p \geq 0$;

(iii) the series is absolutely convergent at every $x \in E^{*}$.

For $x \in E^{*}$ a linear function $\delta_{x}$ on $(E)$ is defined by

$$
\delta_{x}: \phi \mapsto \phi(x), \quad \phi \in(E) .
$$

This is called a white noise delta function.

TheOREm 3.2. $\delta_{x} \in(E)^{*}$ for all $x \in E^{*}$. Moreover,

$$
\left\|\delta_{x}\right\|_{-p} \leq\left\{1-\left(|\tau|_{-p}^{1 / 2}+|x|_{-p}\right)^{2}\right\}^{-1 / 2},
$$

whenever $|\tau|_{-p}^{1 / 2}+|x|_{-p}<1$.

Theorem 3.3 The map $x \mapsto \delta_{x} \in(E)^{*}, x \in E^{*}$, is continuous.

In short, Theorems 3.1-3.3 say that the space of test functionals $(E)$ satisfies the hypotheses (H1)-(H3) introduced in $\S 1$, The proof of Theorem 3.1 being somehow long, it is devided into three steps (Propositions 3.4-3.6) and will be completed in $\S 5$. The estimate (8) in Theorem 3.2 will be sharpened in (33) in $\S 5$.

Proposition 3.4. Let $\tilde{\phi}$ be a continuous function on $E^{*}$. If $\tilde{\phi}(x)=0$ for $\mu$-a.e. $x \in E^{*}$, then $\tilde{\phi}(x)=0$ for all $x \in E^{*}$.

Proof. Note first that $\mu\left(E_{-p}\right)=1$ for $p \geq 1$. In fact, this follows from (A2) and a general result (e.g., [18: Chap. 3]). We prove the assertion by contradiction. Suppose that $\tilde{\phi}\left(x_{0}\right)>0$ for some point $x_{0} \in E^{*}$. Take $p \geq 1$ such that $x_{0} \in E_{-p}$ and consider the restriction of $\tilde{\phi}$ to $E_{-p}$ which is denoted by the same symbol. Obviously, $\tilde{\phi}$ is a continuous function on $E_{-p}$ with $\tilde{\phi}\left(x_{0}\right)>0$. It then follows from the assumption that there exists a non-empty open subset $U \subset E_{-p}$ such that $\mu(U)=0$. We now take a countable subset $\left\{\xi_{k}\right\}_{k=1}^{\infty} \subset H$ such that

$$
E_{-p}=\bigcup_{k=1}^{\infty}\left(U+\xi_{k}\right)
$$

But since the Gaussian measure $\mu$ is quasi-invariant under translations by $H$, we have $\mu\left(U+\xi_{k}\right)=0$ and therefore $\mu\left(E_{-p}\right)=0$. This is contradiction. Q.E.D.

We now introduce two basic constant numbers in white noise calculus: 


$$
\delta=\left\|A^{-1}\right\|_{\mathrm{HS}}=\left(\sum_{j=0}^{\infty} \lambda_{j}^{-2}\right)^{1 / 2}, \quad \rho=\left\|A^{-1}\right\|=\lambda_{0}^{-1},
$$

where $\left\|A^{-1}\right\|_{\mathrm{HS}}$ and $\left\|A^{-1}\right\|$ stand for the Hilbert-Schmidt norm and the operator norm of $A^{-1}$, respectively. These are frequently used together with the obvious inequalities:

$$
0<\rho<1, \quad \rho<\delta
$$

which follow from (A3).

Again by $|\cdot|_{p}$ we denote the norm of the Hilbert space $E_{p}^{\otimes n}, p \in \mathbf{R}$. Then, in view of (1) we obtain

$$
\begin{aligned}
|\omega|_{p} & =\left|\left(A^{\otimes n}\right)^{p} \omega\right|_{0} \\
& =\left(\sum_{j_{1}, \cdots, j_{n}=0}^{\infty} \lambda_{j_{1}}^{2 p} \cdots \lambda_{j_{n}}^{2 p}\left\langle\omega, e_{j_{1}} \otimes \cdots \otimes e_{j_{n}}\right\rangle^{2}\right)^{1 / 2}, \quad \omega \in E_{p}^{\otimes n} .
\end{aligned}
$$

Note also that

$$
|\omega|_{p} \leq \rho^{n}|\omega|_{p+1}, \quad \omega \in E_{p}^{\otimes n}, \quad p \in \mathbf{R},
$$

and therefore

$$
\lim _{p \rightarrow \infty}|F|_{-p}=0, \quad F \in\left(E^{\otimes n}\right)^{*} .
$$

Proposition 3.5. For $n=0,1,2, \ldots$ let $E_{\mathbf{C}}^{\hat{\otimes} n}$ and assume $\sum_{n=0}^{\infty} n !\left|f_{n}\right|_{p}^{2}<\infty$ for all $p \geq 0$. Then the series

$$
\sum_{n=0}^{\infty}\left\langle: x^{\otimes n}:, f_{n}\right\rangle
$$

converges absolutely at each $x \in E^{*}$.

Proof. By definition (3) we have $\tau=\sum_{j=0}^{\infty} e_{j} \otimes e_{j}$ and $|\tau|_{-p}^{2}=$ $\sum_{j=0}^{\infty} \lambda_{j}^{-4 p}<\infty$ whenever $p>1 / 2$. We next note the inequality:

$$
\left|: x^{\otimes n}:\right|_{-p} \leq \sqrt{n !}\left(|\tau|_{-p}^{1 / 2}+|x|_{-p}\right)^{n},
$$

which follows from the well-known identity:

$$
: x^{\otimes n}:=\sum_{k=0}^{[n / 2]} \frac{(-1)^{k} n !}{(n-2 k) ! k ! 2^{k}} \tau^{\hat{\otimes} k} \widehat{\otimes} x^{\otimes(n-2 k)},
$$


and an obvious inequality:

$$
\frac{1}{k ! 2^{k}} \leq \frac{\sqrt{n !}}{(2 k) !}, \quad 0 \leq k \leq\left[\frac{n}{2}\right]
$$

Now let $x \in E^{*}$ be fixed. It follows from (13) that

$$
\begin{aligned}
\sum_{n=0}^{\infty}\left|\left\langle: x^{\otimes n}:, f_{n}\right\rangle\right| & \leq \sum_{n=0}^{\infty}\left|: x^{\otimes n}:\right|_{-p}\left|f_{n}\right|_{p} \\
& \leq \sum_{n=0}^{\infty} \sqrt{n !}\left(|\tau|_{-p}^{1 / 2}+|x|_{-p}\right)^{n}\left|f_{n}\right|_{p} \\
& \leq\left(\sum_{n=0}^{\infty} n !\left|f_{n}\right|_{p}^{2}\right)^{1 / 2}\left(\sum_{n=0}^{\infty}\left(|\tau|_{-p}^{1 / 2}+|x|_{-p}\right)^{2 n}\right)^{1 / 2} .
\end{aligned}
$$

In view of (12) we choose $p>1 / 2$ such that $|\tau|_{-p}^{1 / 2}+|x|_{-p}<1$. Then (15) becomes

$$
\sum_{n=0}^{\infty}\left|\left\langle: x^{\otimes n}:, f_{n}\right\rangle\right| \leq\left(\sum_{n=0}^{\infty} n !\left|f_{n}\right|_{p}^{2}\right)^{1 / 2}\left\{1-\left(|\tau|_{-p}^{1 / 2}+|x|_{-p}\right)^{2}\right\}^{-1 / 2}<\infty
$$

as desired.

Q.E.D.

We now go back to the proof of Theorem 3.1. According to Proposition 2.2 a given $\phi \in(E)$ admits an expression:

$$
\phi(x)=\sum_{n=0}^{\infty}\left\langle: x^{\otimes n}:, f_{n}\right\rangle, \quad x \in E^{*},
$$

where $f_{n} \in E_{\mathbf{C}}^{\hat{\otimes} n}$ for $n=0,1,2, \ldots$ and $\sum_{n=0}^{\infty} n !\left|f_{n}\right|_{p}^{2}<\infty$ for all $p \geq 0$. The series converges in $L^{2}$-sense. On the other hand, it follows from Proposition 3.5 that

$$
\tilde{\phi}(x) \equiv \sum_{n=0}^{\infty}\left\langle: x^{\otimes n}:, f_{n}\right\rangle
$$

converges at every point $x \in E^{*}$. Therefore, $\phi(x)=\tilde{\phi}(x)$ for $\mu$-a.e. $x \in E^{*}$. Since the uniqueness of a continuous version follows from Proposition 3.4, the proof of Theorem 3.1 is completed by the following

Proposition 3.6. For $n=0,1,2, \ldots$ let $f_{n} \in E_{\mathbf{C}}^{\hat{\otimes} n}$ and assume $\sum_{n=0}^{\infty} n !\left|f_{n}\right|_{p}^{2}$ $<\infty$ for all $p \geq 0$. Then, $\tilde{\phi}$ defined in (17) is a continuous function on $E^{*}$.

It is much simpler to show that the restriction of $\tilde{\phi}$ to $E_{-p}$ is continuous with respect to the norm $|\cdot|_{-p}$. However, this is not enough to assert the continuity of $\tilde{\phi}$ 
with respect to the strong dual topology of $E^{*}$, because the inductive system $\left\{E_{-p}\right\}_{p \geq 0}$ is not strict.

Proposition 3.6 will be shown in Proposition 5.4 together with a precise estimate of $|\tilde{\phi}(x)-\tilde{\phi}(y)|$. While, the proof of Theorem 3.2 has been already established during the proof of Proposition 3.5. The estimate (8) follows immediately from (16).

\section{Defining seminorms for the strong dual $E^{*}$}

Following [18: Chap. 3] we introduce a set of defining Hilbertian seminorms of $E^{*}$. Let $\mathscr{C}$ be the set of sequences $C=\left(C_{p}\right)_{p=0}^{\infty}$ such that $C_{0} \geq C_{1} \geq \cdots>0$. For $C \in \mathscr{C}$ we put

$$
\square \xi \square_{C}^{2}=\sum_{p=0}^{\infty} C_{p}^{2}|\xi|_{p}^{2}, \quad \xi \in E,
$$

though possibly $\left[\xi \square_{C}=\infty\right.$. Then $E(C)=\left\{\xi \in E ; \square \xi \square_{C}<\infty\right\}$ becomes a Hilbert space with norm $\left[\cdot \square_{C}\right.$. We put

$$
|x|_{C}=\sup \left\{|\langle x, \xi\rangle| ;[\xi]_{C} \leq 1, \xi \in E\right\}, \quad x \in E^{*} .
$$

Obviously, $\mid \cdot I_{C}$ is a Hilbertian seminorm on $E^{*}$ though it is not necessarily a norm. Note also that for any $C \in \mathscr{C}$

$$
|\langle x, \xi\rangle| \leq|x|_{C} \square \xi \square_{C}, \quad x \in E^{*}, \quad \xi \in E,
$$

though $\square \xi \square_{c}=\infty$ can happen.

Lemma 4.1. $\left\{|\cdot|_{C}\right\}_{C \in \mathscr{C}}$ is a set of defining Hilbertian seminorms of $E^{*}$.

Proof. The strong dual topology of $E^{*}$ is defined by the seminorms

$$
x \mapsto \sup \{|\langle x, \xi\rangle| ; \xi \in B\}, \quad x \in E^{*},
$$

where $B$ runs over all bounded subsets of $E$. It is therefore sufficient to show that for any bounded subset $B \subset E$ there is $C \in \mathscr{C}$ such that $B \subset\{\xi \in E ; \square \xi \square]_{C}$ $\leq 1\}$. But this is easily verified.

Q.E.D.

Lemma 4.2. For $C=\left(C_{p}\right)_{p=0}^{\infty} \in \mathscr{C}$ it holds that

$$
|x|_{c}^{2}=\sum_{j=0}^{\infty}\left\langle x, e_{j}\right\rangle^{2} \square e_{j} \square_{c}^{-2}=\sum_{j=0}^{\infty}\left\langle x, e_{j}\right\rangle^{2}\left(\sum_{p=0}^{\infty} C_{p}^{2} \lambda_{j}^{2 p}\right)^{-1}, \quad x \in E^{*}
$$


Proof. Recall that $\left\{e_{j}\right\}_{j=0}^{\infty}$ is an orthogonal basis for all $E_{p}, p \geq 0$. In view of (18) we see that $\left\{\square e_{j} \square_{c}^{-1} e_{j}\right\}_{j=0}^{\infty}$ is an orthonormal basis for $E(C)$ and also for $E(C)^{*}$, where we understand $\square e_{j} \square_{c}^{-1} e_{j}=0$ if $\square e_{j} \square_{c}=\infty$. Then the assertion follows from Fourier expansion $x=\sum_{j=0}^{\infty}\left\langle x, e_{j}\right\rangle e_{j}$, which converges in $E^{*}$.

Q.E.D.

It is noted that

$$
E^{\otimes n}=\bigcap_{p \geq 0} E_{p}^{\otimes n} \cong \underset{p \rightarrow \infty}{\operatorname{proj} \lim } E_{p}^{\otimes n}, \quad\left(E^{\otimes n}\right)^{*}=\underset{p \geq 0}{\bigcup} E_{-p}^{\otimes n} \cong \underset{p \rightarrow \infty}{\operatorname{ind} \lim } E_{-p}^{\otimes n} .
$$

Therefore the topology of $\left(E^{\otimes n}\right)^{*}$ is defined in a similar way. Namely, for $C \in \mathscr{C}$ put

$$
\square \omega \square_{C}^{2}=\sum_{p_{1}, \cdots, p_{n}=0}^{\infty} C_{p_{1}}^{2} \cdots C_{p_{n}}^{2}|\omega|_{p_{1}, \cdots, p_{n}}^{2}, \quad \omega \in E^{\otimes n}
$$

where

$$
\begin{aligned}
|\omega|_{p_{1}, \cdots, p_{n}}^{2} & =\left|\left(A^{p_{1}} \otimes \cdots \otimes A^{p_{n}}\right) \omega\right|_{0}^{2} \\
& =\sum_{j_{1}, \cdots, j_{n}=0}^{\infty} \lambda_{j_{1}}^{2 p_{1}} \cdots \lambda_{j_{n}}^{2 p_{n}}\left\langle\omega, e_{j_{1}} \otimes \cdots \otimes e_{j_{n}}\right\rangle^{2},
\end{aligned}
$$

see also (10). Then for $F \in\left(E^{\otimes n}\right)^{*}$ we put

$$
|F|_{C}=\sup \left\{|\langle F, \omega\rangle| ; \square \omega \square_{C} \leq 1, \omega \in E^{\otimes n}\right\} .
$$

It is proved in a similar way to Lemma 4.1 that $\left\{|\cdot|_{C}\right\}_{C \in \mathscr{C}}$ is a set of defining Hilbertian seminorms of $\left(E^{\otimes n}\right)^{*}$. We next note the following

Lemma 4.3. Let $C=\left(C_{p}\right)_{p=0}^{\infty} \in \mathscr{C}$. Then

$$
|F|_{C} \leq C_{p}^{-n}|F|_{-p}, \quad F \in\left(E^{\otimes n}\right)^{*},
$$

though $|F|_{-p}=\infty$ may happen. Moreover, $\mid \cdot I_{c}$ is a cross norm, i.e.,

$$
\left|x_{1} \otimes \cdots \otimes x_{n}\right|_{c}=\left|x_{1}\right|_{c} \cdots\left|x_{n}\right|_{c}, \quad x_{1}, \cdots, x_{n} \in E^{*} \text {. }
$$

Proof. A similar argument as in Lemma 4.1 yields

$$
|F|_{C}^{2}=\sum_{j_{1}, \cdots, j_{n}=0}^{\infty}\left\langle F, e_{j_{1}} \otimes \cdots \otimes e_{j_{n}}\right\rangle^{2} \square e_{j_{1}} \square_{C}^{-2} \cdots \square e_{j_{n}} \square_{C}^{-2} .
$$

It is then obvious that $|\cdot|_{C}$ is a cross norm. Since 


$$
\square e_{j} \square_{c}^{-2}=\left(\sum_{p=0}^{\infty} C_{p}^{2} \lambda_{j}^{2 p}\right)^{-1} \leq C_{p}^{-2} \lambda_{j}^{-2 p}
$$

for any $p \geq 0$, it follows from (23) and (10) that

$$
|F|_{C}^{2} \leq C_{p}^{-2 n} \sum_{j_{1}, \cdots, j_{n}=0}^{\infty} \lambda_{j_{1}}^{-2 p} \cdots \lambda_{j_{n}}^{-2 p}\left\langle F, e_{j_{1}} \otimes \cdots \otimes e_{j_{n}}\right\rangle^{2}=C_{p}^{-2 n}|F|_{-p}^{2}
$$

as desired.

Q.E.D.

\section{Proof of the main results}

For any $C=\left(C_{p}\right)_{p=0}^{\infty} \in \mathscr{C}$ we put

$$
\square \phi \square_{C}^{2}=\sum_{p=0}^{\infty} C_{p}^{2}\|\phi\|_{p}^{2}, \quad \phi \in(E),
$$

though $\left[\phi \square_{c}=\infty\right.$ may happen.

Lemma 5.1. Let $C=\left(C_{p}\right)_{p=0}^{\infty} \in \mathscr{C}$. Then for any $\phi \in(E)$ it holds that

$$
n ! \square f_{n} \square_{C}^{2} \leq C_{0}^{-2} \square \phi \square_{C}^{2}\left(\frac{C_{0}^{2}}{1-\rho^{2}}\right)^{n},
$$

where $\left(f_{n}\right)_{n=0}^{\infty}$ is given as in Proposition 2.1.

Proof. Recall the definition (21) to obtain

$$
\square f_{n} \square_{C}^{2}=\sum_{p_{1}, \cdots, p_{n}=0}^{\infty} C_{p_{1}}^{2} \cdots C_{p_{n}}^{2}\left|f_{n}\right|_{p_{1}, \cdots, p_{n}}^{2}
$$

In view of (10) and (22) we obtain

$$
C_{p_{1}}^{2} \cdots C_{p_{n}}^{2}\left|f_{n}\right|_{p_{1}, \cdots, p_{n}}^{2} \leq C_{0}^{2(n-1)} C_{p}^{2} \rho^{2\left(p-p_{1}\right)+\cdots+2\left(p-p_{n}\right)}\left|f_{n}\right|_{p}^{2},
$$

where $p=\max \left\{p_{1}, \cdots, p_{n}\right\}$. We then see that

$$
\begin{aligned}
\sum_{\max \left\{p_{1}, \cdots, p_{n}\right\}=p} C_{p_{1}}^{2} \cdots C_{p_{n}}^{2}\left|f_{n}\right|_{p_{1}, \cdots, p_{n}}^{2} & \leq C_{0}^{2(n-1)} C_{p}^{2}\left|f_{n}\right|_{p}^{2}\left(\sum_{q=0}^{p} \rho^{2(p-q)}\right)^{n} \\
& \leq C_{0}^{2(n-1)} C_{p}^{2}\left|f_{n}\right|_{p}^{2}\left(1-\rho^{2}\right)^{-n} .
\end{aligned}
$$

Thereby (25) becomes

$$
\square f_{n} \square_{C}^{2}=\sum_{p=0}^{\infty} \sum_{\max \left\{p_{1}, \cdots, p_{n}\right\}=p} C_{p_{1}}^{2} \cdots C_{p_{n}}^{2}\left|f_{n}\right|_{p_{1}, \cdots, p_{n}}^{2}
$$




$$
\leq C_{0}^{-2}\left(\frac{C_{0}^{2}}{1-\rho^{2}}\right)^{n} \sum_{p=0}^{\infty} C_{p}^{2}\left|f_{n}\right|_{p}^{2}
$$

Finally with an obvious inequality: $n !\left|f_{n}\right|_{p}^{2} \leq\|\phi\|_{p}^{2}$, which follows from (7), we obtain

$$
n ! \square f_{n} \square_{C}^{2} \leq C_{0}^{-2}\left(\frac{C_{0}^{2}}{1-\rho^{2}}\right)^{n} \sum_{p=0}^{\infty} C_{p}^{2}\|\phi\|_{p}^{2}=C_{0}^{-2} \square \phi \square_{C}^{2}\left(\frac{C_{0}^{2}}{1-\rho^{2}}\right)^{n} .
$$

This completes the proof.

Q.E.D.

LEMMA 5.2. For $k=0,1,2, \ldots$ it holds that

$$
\sum_{n=0}^{\infty} \frac{(n+k) !}{n ! n !} t^{n} \leq(t+k)^{k} e^{t}, \quad t \geq 0
$$

Proof. We put

$$
P_{k}(t)=e^{-t} \sum_{n=0}^{\infty} \frac{(n+k) !}{n ! n !} t^{n}
$$

As is easily shown, $P_{k}(t)$ is a polynomial of degree $k$. Actually, it is related to the Laguerre polynomial (e.g. [6: Appendix]) as $P_{k}(t)=k ! L_{k}(-t)$. We thus put $P_{k}(t)$ $=\sum_{l=0}^{k} a_{k l} t^{l}$. Then, by induction we may prove

$$
0 \leq a_{k l} \leq\left(\begin{array}{l}
k \\
l
\end{array}\right) k^{k-l}, \quad 0 \leq l \leq k
$$

from which the assertion follows immediately.

Q.E.D.

LEMma 5.3. Put

$$
\lambda(z, w)=\sum_{k=0}^{\infty} \frac{(z+k)^{k+1} w^{k}}{k !} .
$$

Then the series converges in $\mathbf{C} \times\left\{|\omega|<e^{-1}\right\}$ and $\lambda(z, w)$ becomes a holomorphic function in two variables.

Proof. We only need to apply Cauchy-Hadamard formula.

Q.E.D.

Assuming that $C=\left(C_{p}\right)_{p=0}^{\infty} \in \mathscr{C}$ satisfies the condition $C_{0}^{2}<1-\rho^{2}$, we put 


$$
\Lambda_{C}(z, w)=\frac{2}{\sqrt{1-\rho^{2}-C_{0}^{2}}} \exp \left(\frac{z^{2}}{2}\right) \lambda\left(\frac{z^{2}}{2}+1, \frac{C_{0}^{2} w}{1-\rho^{2}}\right) .
$$

Obviously, $\Lambda_{C}(z, w)$ is holomorphic in $\mathbf{C} \times\left\{|w|<e^{-1} C_{0}^{-2}\left(1-\rho^{2}\right)\right\}$. With these notations we have

Proposition 5.4. For $n=0,1,2, \ldots$ let $f_{n} \in E_{\mathbf{C}}^{\hat{\otimes}^{n}}$ and assume $\sum_{n=0}^{\infty} n !\left|f_{n}\right|_{p}^{2}$ $<\infty$ for all $p \geq 0$. Put

$$
\tilde{\phi}(x)=\sum_{n=0}^{\infty}\left\langle: x^{\otimes n}:, f_{n}\right\rangle, \quad x \in E^{*} .
$$

If $C=\left(C_{p}\right)_{p=0}^{\infty} \in \mathscr{C}$ satisfies

$$
C_{0}^{2}<1-p^{2} \quad \text { and } \quad C_{0}^{2}|\tau|_{c}<\frac{1-\rho^{2}}{e},
$$

then

$$
\left.|\tilde{\phi}(x)-\tilde{\phi}(y)| \leq|x-y|_{C} \square \tilde{\phi}\right]_{C} \Lambda_{C}\left(|x|_{C}+|y|_{c},|\tau|_{C}\right)
$$

for all $x, y \in E^{*}$. In particular, $\tilde{\phi}$ is a continuous function on $E^{*}$.

Proof. Let $x, y \in E^{*}$ and suppose $C=\left(C_{p}\right)_{p=0}^{\infty} \in \mathscr{C}$ satisfies (26). Then, in view of (14), (20) and Lemma 4.3 we observe

$$
\begin{aligned}
\mid\left\langle: x^{\otimes n}\right. & \left.:-: y^{\otimes n}:, f_{n}\right\rangle \mid \\
& \leq \sum_{k=0}^{[n / 2]} \frac{n !}{(n-2 k) ! k ! 2^{k}}\left|\left\langle\tau^{\hat{\otimes} k} \widehat{\otimes}\left(x^{\otimes(n-2 k)}-y^{\otimes(n-2 k)}\right), f_{n}\right\rangle\right| \\
& \left.\leq \sum_{k=0}^{[n / 2]} \frac{n !}{(n-2 k) ! k ! 2^{k}}|\tau|_{C}^{k}\left|x^{\otimes(n-2 k)}-y^{\otimes(n-2 k)}\right|_{C} \square f_{n} \square\right]_{C} .
\end{aligned}
$$

Using an obvious inequality:

$$
\left|x^{\otimes m}-y^{\otimes m}\right|_{C} \leq|x-y|_{C}\left(|x|_{C}+|y|_{C}\right)^{m-1}, \quad m \geq 1
$$

and summing up both sides of (28) with $n$, we obtain

$$
\begin{aligned}
|\tilde{\phi}(x)-\tilde{\phi}(y)| \leq|x-y|_{C} \sum_{k=0}^{\infty} \frac{|\tau|_{C}^{k}}{k ! 2^{k}} \sum_{n=0}^{\infty} \frac{(n+2 k+1) !}{(n+1) !} \\
\left.\times\left(|x|_{C}+|y|_{C}\right)^{n} \square f_{n+2 k+1} \square\right]_{C} .
\end{aligned}
$$

Applying the Schwarz inequality, we have 


$$
\begin{aligned}
& \sum_{n=0}^{\infty} \frac{(n+2 k+1) !}{(n+1) !}\left(|x|_{c}+|y|_{c}\right)^{n} \square f_{n+2 k+1} \square_{c} . \\
& \quad \leq\left(\sum_{n=0}^{\infty}(n+2 k+1) ! \square f_{n+2 k+1} \square_{c}^{2}\right)^{1 / 2} \\
& \quad \times\left(\sum_{n=0}^{\infty} \frac{(n+2 k+1) !}{(n+1) !(n+1) !}\left(|x|_{c}+|y|_{c}\right)^{2 n}\right)^{1 / 2}
\end{aligned}
$$

We now estimate the last two series. By Lemma 5.1 we have

$$
\begin{aligned}
\sum_{n=0}^{\infty}(n+2 k+1) ! \square f_{n+2 k+1} \square_{C}^{2} & \leq C_{0}^{-2} \square \tilde{\phi} \square_{C}^{2} \sum_{n=0}^{\infty}\left(\frac{C_{0}^{2}}{1-\rho^{2}}\right)^{n+2 k+1} \\
& =\frac{\square \tilde{\phi} \square_{C}^{2}}{1-\rho^{2}-C_{0}^{2}}\left(\frac{C_{0}^{2}}{1-\rho^{2}}\right)^{2 k}
\end{aligned}
$$

where we used the assumption $C_{0}^{2}<1-\rho^{2}$. On the other hand, application of Lemma 5.2 yields

$$
\begin{aligned}
& \sum_{n=0}^{\infty} \frac{(n+2 k+1) !}{(n+1) !(n+1) !}\left(|x|_{C}+|y|_{C}\right)^{2 n} \\
& \quad \leq \sum_{n=0}^{\infty} \frac{(n+2 k+2) !}{n ! n !}\left(|x|_{C}+|y|_{C}\right)^{2 n} \\
& \quad \leq\left\{\left(|x|_{C}+|y|_{C}\right)^{2}+2 k+2\right\}^{2 k+2} \exp \left\{\left(|x|_{C}+|y|_{C}\right)^{2}\right\}
\end{aligned}
$$

Therefore (30) is estimated by (31) and (32) as

$$
\begin{gathered}
\sum_{n=0}^{\infty} \frac{(n+2 k+1) !}{(n+1) !}\left(|x|_{c}+|y|_{c}\right)^{n} \square f_{n+2 k+1} \square_{C} \\
\leq \frac{2 \square \tilde{\phi} \square_{C}}{\sqrt{1-\rho^{2}-C_{0}^{2}}}\left(\frac{2 C_{0}^{2}}{1-\rho^{2}}\right)^{k} \exp \left(\frac{\left(|x|_{C}+|y|_{c}\right)^{2}}{2}\right) \\
\times\left\{\frac{\left(|x|_{C}+|y|_{c}\right)^{2}}{2}+k+1\right\}^{k+1} .
\end{gathered}
$$

Consequently, (29) becomes

$$
\begin{aligned}
|\tilde{\phi}(x)-\tilde{\phi}(y)| \leq \frac{2 \square \tilde{\phi} \square_{C}|x-y|_{C}}{\sqrt{1-\rho^{2}-C_{0}^{2}}} \exp \left(\frac{\left(|x|_{C}+|y|_{C}\right)^{2}}{2}\right) \\
\quad \times \sum_{k=0}^{\infty} \frac{1}{k !}\left(\frac{C_{0}^{2}|\tau|_{C}}{1-\rho^{2}}\right)^{k}\left\{\frac{\left(|x|_{C}+|y|_{C}\right)^{2}}{2}+k+1\right\}^{k+1}
\end{aligned}
$$




$$
\begin{aligned}
& =\frac{2\left[\tilde{\phi} \square_{C}|x-y|_{C}\right.}{\sqrt{1-\rho^{2}-C_{0}^{2}}} \exp \left(\frac{\left(|x|_{C}+|y|_{C}\right)^{2}}{2}\right) \\
& \quad \times \lambda\left(\frac{\left(|x|_{C}+|y|_{C}\right)^{2}}{2}+1, \frac{C_{0}^{2}|\tau|_{C}}{1-\rho^{2}}\right) \\
& =|x-y|_{C} \square \tilde{\phi} \square_{C} \Lambda_{C}\left(|x|_{C}+|y|_{C},|\tau|_{C}\right) .
\end{aligned}
$$

This completes the proof of (27). Take $C=\left(C_{p}\right)_{p=0}^{\infty} \in \mathscr{C}$ with the properties (26) (such a $C$ exists certainly). Since $z \mapsto \Lambda_{C}\left(z,|\tau|_{C}\right)$ is continuous (in fact, analytic), we conclude from (27) that $\tilde{\phi}$ is continuous on $E^{*}$.

Q.E.D.

As was already explained in $\S 3$, Proposition 5.4 completes the proof of the continuous version theorem (Theorem 3.1). Theorem 3.2 was proved at the end of $\S 3$. We now give

Proof of Theorem 3.3. Since $(E)^{*}$ is constructed in a similar way to $E^{*}$ by the standard construction, the topology of $(E)^{*}$ is defined by the Hilbertian seminorms:

$$
\Phi \mapsto \sup \left\{|\langle\Phi, \phi\rangle| ; \square \phi \square_{C} \leq 1\right\}, \quad \Phi \in(E)^{*},
$$

where $\square \phi \square_{C}$ is defined as in $(24)$ and $C$ runs over $\mathscr{C}$. While, it follows from Proposition 5.4 that

$$
\lim _{y \rightarrow x} \sup \left\{\left|\left\langle\delta_{x}-\delta_{y}, \phi\right\rangle\right| ; \mid[\phi]_{c} \leq 1\right\}=0,
$$

for all $C=\left(C_{p}\right)_{p=0}^{\infty} \in \mathscr{C}$ satisfying the conditions in (26). It is therefore sufficient to show that all $C \in \mathscr{C}$ with (26) constitute a set of defining seminorms of $E^{*}$. Note that $|x|_{C} \leq|x|_{C^{\prime}}$ for any $x \in E^{*}$ if $C^{\prime} \leq C$, namely, if $C_{p}^{\prime} \leq C_{p}$ for all $p=$ $0,1,2, \ldots$ Thus it is sufficient to show that for a given $C \in \mathscr{C}$ there is $C^{\prime} \in \mathscr{C}$ with (26) such that $C^{\prime} \leq C$. Choose $q \geq 0$ such that $|\tau|_{-p}<e^{-1}\left(1-\rho^{2}\right)$. Define $C^{\prime}=\left(C_{p}^{\prime}\right)_{p=0}^{\infty} \in \mathscr{C}$ by

$$
\left\{\begin{array}{l}
0<C_{0}^{\prime}=\cdots=C_{q}^{\prime}<\min \left\{|\tau|_{-q}, C_{q}, \sqrt{1-\rho^{2}}\right\}, \\
C_{p}^{\prime}=\min \left\{C_{p-1}^{\prime}, C_{p}\right\}, \quad p>q .
\end{array}\right.
$$

Then, by construction, $C^{\prime} \leq C$ and $C_{0}^{\prime 2}<1-\rho^{2}$. Moreover, since $|\tau|_{C^{\prime}} \leq$ $C_{q}^{\prime-2}|\tau|_{-q}$ by Lemma 4.3 , we have

$$
C_{0}^{\prime 2}|\tau|_{C^{\prime}} \leq C_{0}^{\prime 2} C_{q}^{\prime-2}|\tau|_{-q}=|\tau|_{-q} \leq \frac{1-\rho^{2}}{e}
$$


This completes the proof.

Q.E.D.

Remark. By a similar (but much simpler) computation as in the proof of Propositon 5.4, we obtain a somehow better estimate of a white noise delta function $\delta_{x}, x \in E^{*}$. Let $\phi \in(E)$ be given by

$$
\phi(x)=\sum_{n=0}^{\infty}\left\langle: x^{\otimes n}:, f_{n}\right\rangle, \quad x \in E^{*},
$$

as usual. Then, for $p \geq 0$ we have

$$
\begin{aligned}
|\phi(x)| & \leq \sum_{n=0}^{\infty}\left|\left\langle: x^{\otimes n}:, f_{n}\right\rangle\right| \\
& \leqq\|\phi\|_{p} \exp \left(\frac{|x|_{-p}^{2}}{2}\right) \sum_{k=0}^{\infty} \frac{|\tau|_{-p}^{k}}{k !}\left(\frac{|x|_{-p}^{2}}{2}+k\right)^{k},
\end{aligned}
$$

and therefore,

$$
\left\|\delta_{x}\right\|_{-p} \leq \exp \left(\frac{|x|_{-p}^{2}}{2}\right) \sum_{k=0}^{\infty} \frac{|\tau|_{-p}^{k}}{k !}\left(\frac{|x|_{-p}^{2}}{2}+k\right)^{k}
$$

The last series converges whenever $|\tau|_{-p}<e^{-1}$ and $|x|_{-p}<\infty$. This condition may be compared with $|\tau|_{-p}^{1 / 2}+|x|_{-p}<1$ in Theorem 3.2 .

\section{Tensor product}

The standard construction of a Gelfand triple is well suited to tensor products. We begin with the following

Proposition 6.1. For $i=1,2$ let $A_{\imath}$ be a standard operator on $H_{1}$ and let $E_{i} \subset$ $H_{i} \subset E_{i}^{*}$ be the Gelfand triple constructed in the standard manner. Then, $A_{1} \otimes A_{2}$ is a standard operator on $H_{1} \otimes H_{2}$ with domain $\operatorname{Dom}\left(A_{1} \otimes A_{2}\right)=\operatorname{Dom}\left(A_{1}\right) \otimes_{\text {alg }}$ $\operatorname{Dom}\left(A_{2}\right)$ and the Gelfand triple obtained from $A_{1} \otimes A_{2}$ is given by $E_{1} \otimes E_{2} \subset H_{1} \otimes$ $H_{2} \subset\left(E_{1} \otimes E_{2}\right)^{*}$.

Proof. It follows from Theorem 1.1 that $E_{i}=$ proj $\lim _{p \rightarrow \infty} E_{i p}, i=1,2$, where $E_{i p}$ is the Hilbert space obtained by completing $E_{i}$ with respect to the norm $|\xi|_{p}=\left|A_{i}^{p} \xi\right|_{0}, \xi \in E_{i}$. Then a simple observation implies that

$$
E_{1} \otimes E_{2}=\underset{p, q \rightarrow \infty}{\operatorname{proj} \lim } E_{1 p} \otimes E_{2 q}=\underset{p \rightarrow \infty}{\operatorname{proj} \lim } E_{1 p} \otimes E_{2 p} .
$$

On the other hand, it is easily verified that $A_{1} \otimes A_{2}$ is a standard operator on 
$H_{1} \otimes H_{2}$. Let $F$ be the nuclear Fréchet space constructed from $A_{1} \otimes A_{2}$ in the standard manner. Then, $F=\operatorname{proj} \lim _{p \rightarrow \infty} F_{p}$ with $F_{p}$ being the completion of $F$ with respect to the norm $|\zeta|_{p}=\left|\left(A_{1} \otimes A_{2}\right)^{p} \zeta\right|_{0}$. Note here that $F_{p}=E_{1 p} \otimes E_{2 p}$. It then follows from (34) that $F=E_{1} \otimes E_{2}$.

Q.E.D.

Proposition 6.2. For $i=1,2$ let $\Omega_{\imath}$ be a topological space with a Borel measure $\nu_{i}$ and let $A_{i}$ be a standard operator on $L^{2}\left(\Omega_{\imath}, \nu_{i} ; \mathbf{R}\right)$. Then

$$
\mathscr{S}_{A_{1} \otimes A_{2}}\left(\Omega_{1} \times \Omega_{2}\right)=\mathscr{S}_{A_{1}}\left(\Omega_{1}\right) \otimes \mathscr{S}_{A_{2}}\left(\Omega_{2}\right)
$$

under the identification: $L^{2}\left(\Omega_{1} \times \Omega_{2}, \nu_{1} \times \nu_{2} ; \mathbf{R}\right)=L^{2}\left(\Omega_{1}, \nu_{1} ; \mathbf{R}\right) \otimes L^{2}\left(\Omega_{2}, \nu_{2} ; \mathbf{R}\right)$.

Proof. Immediate from Proposition 6.1.

Q.E.D.

Proposition 6.3. Let notations and assumptions be the same as in Proposition 6.2. If both $\mathscr{S}_{A_{1}}\left(\Omega_{1}\right)$ and $\mathscr{S}_{A_{2}}\left(\Omega_{2}\right)$ satisfy the hypotheses $(\mathrm{H} 1)-(\mathrm{H} 3)$, so does $\oiint_{A_{1} \otimes A_{2}}\left(\Omega_{1} \times \Omega_{2}\right)$.

Proof. For $\zeta \in \mathscr{A}_{A_{1} \otimes A_{2}}\left(\Omega_{1} \times \Omega_{2}\right)=\oiint_{A_{1}}\left(\Omega_{1}\right) \otimes \oiint_{A_{2}}\left(\Omega_{2}\right)$ we put

$$
\tilde{\zeta}\left(\omega_{1}, \omega_{2}\right)=\left\langle\delta_{\omega_{1}} \otimes \delta_{\omega_{2}}, \zeta\right\rangle, \quad \omega_{1} \in \Omega_{1}, \quad \omega_{2} \in \Omega_{2} .
$$

Then $\tilde{\zeta}$ is a continuous function on $\Omega_{1} \times \Omega_{2}$ because of (H3) and the fact that

$$
x, y \mapsto x \otimes y \in \mathscr{S}_{A_{1}}^{*}\left(\Omega_{1}\right) \otimes \mathscr{S}_{A_{2}}^{*}\left(\Omega_{2}\right), \quad x \in \mathscr{S}_{A_{1}}^{*}\left(\Omega_{1}\right), \quad y \in \mathscr{\bigotimes}_{A_{2}}^{*}\left(\Omega_{2}\right),
$$

is continuous. Take an approximating sequence $\left\{\zeta_{n}\right\}_{n=1}^{\infty} \subset \mathscr{A}_{A_{1}}\left(\Omega_{1}\right) \bigotimes_{\text {alg }} \mathscr{\bigotimes}_{A_{2}}\left(\Omega_{2}\right)$ such that

$$
\lim _{n \rightarrow \infty}\left|\zeta_{n}-\zeta\right|_{p}=0 \text { for all } p \geq 0
$$

Then

$$
\begin{aligned}
\left|\zeta_{n}-\tilde{\zeta}\right|_{0}^{2} & =\int_{\Omega_{1} \times \Omega_{2}}\left|\zeta_{n}\left(\omega_{1}, \omega_{2}\right)-\tilde{\zeta}\left(\omega_{1}, \omega_{2}\right)\right|^{2} \nu_{1}\left(d \omega_{1}\right) \nu_{2}\left(d \omega_{2}\right) \\
& =\int_{\Omega_{1} \times \Omega_{2}}\left|\left\langle\delta_{\omega_{1}} \otimes \delta_{\omega_{2}}, \zeta_{n}-\zeta\right\rangle\right|^{2} \nu_{1}\left(d \omega_{1}\right) \nu_{2}\left(d \omega_{2}\right) \\
& \leq\left|\zeta_{n}-\zeta\right|_{1}^{2} \int_{\Omega_{1}}\left|\delta_{\omega_{1}}\right|_{-1}^{2} \nu_{1}\left(d \omega_{1}\right) \int_{\Omega_{2}}\left|\delta_{\omega_{2}}\right|_{-2}^{2} \nu_{2}\left(d \omega_{2}\right)
\end{aligned}
$$

Note that

$$
\int_{\Omega_{i}}\left|\delta_{\omega_{i}}\right|_{-2}^{2} \nu_{i}\left(d \omega_{i}\right)=\sum_{j=0}^{\infty} \lambda_{i j}^{-2} \equiv \delta_{i}^{2}<\infty, \quad i=1,2
$$


which is immediate from the identity:

$$
\left|\delta_{\omega_{i}}\right|_{-1}^{2}=\sum_{j=0}^{\infty}\left\langle\delta_{\omega_{i}}, e_{i j}\right\rangle^{2} \lambda_{i j}^{-2}=\sum_{j=0}^{\infty} e_{i j}\left(\omega_{i}\right)^{2} \lambda_{i j}^{-2}, \quad i=1,2 .
$$

It then follows from (36) and (37) that

$$
\left|\zeta_{n}-\tilde{\zeta}\right|_{0}^{2} \leq \delta_{1}^{2} \delta_{2}^{2}\left|\zeta_{n}-\zeta\right|_{1}^{2}
$$

Hence, we see from (35) and (38) that

$$
\begin{aligned}
|\zeta-\tilde{\zeta}|_{0} & \leq\left|\zeta-\zeta_{n}\right|_{0}+\left|\zeta_{n}-\tilde{\zeta}\right|_{0} \\
& \leq\left|\zeta-\zeta_{n}\right|_{0}+\delta_{1} \delta_{2}\left|\zeta_{n}-\zeta\right|_{1} \\
& \rightarrow 0 \text { as } n \rightarrow \infty .
\end{aligned}
$$

Namely, $\zeta\left(\omega_{1}, \omega_{2}\right)=\tilde{\zeta}\left(\omega_{1}, \omega_{2}\right)$ for $\nu_{1} \times \nu_{2}$-a.e. $\left(\omega_{1}, \omega_{2}\right) \in \Omega_{1} \times \Omega_{2}$. This proves (H1). The properties (H2) and (H3) are now immediate.

Q.E.D.

In view of Theorems 3.1-3.3 and Proposition 6.3 we conclude the following

THEOREM 6.4. Any function in the $n$-fold tensor product $(E) \otimes \cdots \otimes(E)$ is continuous on the product space $E^{*} \times \cdots \times E^{*}$ (n times), or more precisely, admits a unique continuous version with respect to the product measure $\mu \times \cdots \times \mu$ ( $n$ times), $n \geq 1$.

\section{Appendix. A sufficient condition for the hypotheses (H1)-(H3)}

We shall give a sufficient condition for (H1)-(H3) from a different viewpoint.

Proposition. Let $\Omega$ be a topological space with a Borel measure $\nu$ and let $A$ be a standard operator on $H=L^{2}(\Omega, \nu ; \mathbf{R})$ with eigenfunctions $\left\{e_{\jmath}\right\}_{{ }_{j}=0}^{\infty}$ and eigenvalues $\left\{\lambda_{j}\right\}_{j=0}^{\infty}$ satisfying (S1) and (S2). Assume the following three conditions:

(i ) $\nu(U)>0$ for any non-empty open subset $U \subset \Omega$;

(ii) every $e_{j}$ is a continuous function on $\Omega$;

(iii) $\Omega$ admits an open covering $\Omega=\cup_{\gamma} \Omega_{\gamma}$ with the property that for each $\gamma$ there exists $\alpha(\gamma) \geq 0$ such that

$$
M_{r} \equiv \sup \left\{\lambda_{j}^{-\alpha(\gamma)}\left|e_{j}(\omega)\right| ; \omega \in \Omega_{r}, j=0,1,2, \ldots\right\}<\infty .
$$

Then $\mathscr{\oiint}_{A}(\Omega)$ satisfies $(\mathrm{H} 1)-(\mathrm{H} 3)$. Moreover, $\tilde{\phi}$ is given by the absolutely convergent series: 


$$
\tilde{\phi}(\omega)=\sum_{j=0}^{\infty}\left\langle\phi, e_{j}\right\rangle e_{j}(\omega)
$$

Proof. By Lemma 1.2 we may assume $r=1$ in (S2). We first show that for $\phi$ $\in \mathscr{\bigotimes}_{A}(\Omega)$ the series (39) converges absolutely at any $\omega \in \Omega$. Choosing $\Omega_{\gamma}$ containing $\omega$, we observe

$$
\begin{aligned}
\sum_{j=0}^{\infty}\left|\left\langle\varphi, e_{j}\right\rangle e_{j}(\omega)\right| & \leq M_{r} \sum_{j=0}^{\infty} \lambda_{j}^{\alpha(r)}\left|\left\langle\phi, e_{j}\right\rangle\right| \\
& \leq M_{r}\left(\sum_{j=0}^{\infty} \lambda_{j}^{2(\alpha(\gamma)+1)}\left\langle\phi, e_{j}\right\rangle^{2}\right)^{1 / 2}\left(\sum_{j=0}^{\infty} \lambda_{j}^{-2}\right)^{1 / 2} .
\end{aligned}
$$

Hence

$$
\sum_{j=0}^{\infty}\left|\left\langle\phi, e_{j}\right\rangle e_{j}(\omega)\right| \leq \delta M_{r}|\phi|_{\alpha(\gamma)+1}, \quad \omega \in \Omega_{r}, \quad \phi \in \mathscr{S}_{A}(\Omega),
$$

where $\delta=\left(\sum_{j=0}^{\infty} \lambda_{j}^{-2}\right)^{1 / 2}<\infty$. This proves that the series (39) converges absolutely at each $\omega \in \Omega$.

For the continuity of $\tilde{\phi}$ we need only to prove that $\tilde{\phi}$ is continuous on $\Omega_{r}$. For $\omega_{1}, \omega_{2} \in \Omega_{r}$, a similar argument as above yields

$$
\left|\tilde{\phi}\left(\omega_{1}\right)-\tilde{\phi}\left(\omega_{2}\right)\right| \leq \sum_{j=0}^{n}\left|\left\langle\phi, e_{j}\right\rangle\right|\left|e_{j}\left(\omega_{1}\right)-e_{j}\left(\omega_{2}\right)\right|+2 M_{\gamma}|\phi|_{\alpha(\gamma)+1}\left(\sum_{j>n} \lambda_{j}^{-2}\right)^{1 / 2} .
$$

Since $e_{j}$ is continuous and $\delta^{2}=\sum_{j=0}^{\infty} \lambda_{j}^{-2}<\infty$ by assumption, the continuity of $\tilde{\phi}$ on $\Omega_{r}$ follows from (41). It is clear that $\phi(\omega)=\tilde{\phi}(\omega)$ for $\nu$-a.e. $\omega \in \Omega$ because the Fourier expansion $\phi=\sum_{j=0}^{\infty}\left\langle\phi, e_{j}\right\rangle e_{j}$ converges in $L^{2}$-sense. We have thus proved (H1).

According to our convention, we do use the symbol $\phi$ for $\tilde{\phi}$ hereafter. The inequality (40) means that the evaluation $\delta_{\omega}: \phi \mapsto \phi(\omega)$ is a continuous linear function on $\mathscr{S}_{A}(\Omega)$. (In fact, $\left|\delta_{\omega}\right|_{-\alpha(\gamma)-1} \leq \delta M_{\gamma}$ for $\omega \in \Omega_{r}$.) Hence, $\delta_{\omega} \in \mathscr{S}_{A}^{*}(\Omega)$ and (H2) holds.

Finally we consider (H3). For a bounded subset $B \subset \mathscr{S}_{A}(\Omega)$ put

$$
|B|_{p}=\sup \left\{|\phi|_{p} ; \phi \in B\right\}
$$

for simplicity. This is always finite. Note that

$$
\sup \left\{\left|\left\langle\phi, e_{j}\right\rangle\right| ; \phi \in B\right\} \leq \sup \left\{|\phi|_{0}\left|e_{j}\right|_{0} ; \phi \in B\right\}=|B|_{0}<\infty .
$$


In view of (41) and (42), for $\omega_{1}, \omega_{2} \in \Omega_{\gamma}$ we have

$$
\begin{aligned}
& \sup \left\{\left|\left\langle\delta_{\omega_{1}}-\delta_{\omega_{2}}, \phi\right\rangle\right| ; \phi \in B\right\} \\
& \leq|B|_{0} \sum_{j=0}^{n}\left|e_{j}\left(\omega_{1}\right)-e_{j}\left(\omega_{2}\right)\right|+2 M_{\gamma}|B|_{\alpha(\gamma)+1}\left(\sum_{j>n} \lambda_{j}^{-2}\right)^{1 / 2} .
\end{aligned}
$$

Hence the map $\omega \mapsto \delta_{\omega} \in \mathscr{S}_{A}^{*}(\Omega)$ is continuous on $\Omega_{\gamma}$ and therefore on $\Omega$. Q.E.D.

\section{REFERENCES}

[1] S. Albeverio, T. Hida, J. Potthoff, M. Röckner and L. Streit, Dirichlet forms in terms of white noise analysis I-Construction and QFT examples, Rev. Math. Phys., 1 (1990), 291-312 ; II-Closability and diffusion processes, ibid., 313-323.

[2] Yu. M. Berezansky and Yu. G. Kondrat'ev, "Spectral Methods in Infinite Dimensional Analysis," Naukova Dumka, Kiev, 1988. (Russian)

[3] M. de Faria, J. Potthoff and L. Streit, The Feynman integrand as a Hida distribution, J. Math. Phys., 32 (1991), 2123-2127.

[ 4 ] I. M. Gelfand and N. Ya. Vilenkin, "Generalized Functions," Vol.4, Academic Press, 1964.

[5] T. Hida, "Analysis of Brownian Functionals," Carleton Math. Lect. Notes No. 13, Carleton University, Ottawa, 1975.

[6] T. Hida, "Brownian Motion," Springer-Verlag, 1980.

[7] T. Hida, H.-H. Kuo and N. Obata, Transformations for white noise functionals, J. Funct. Anal., to appear.

[8] T. Hida, N. Obata and K. Saitô, Infinite dimensional rotations and Laplacians in terms of white noise calculus, Nagoya Math. J., 128 (1992), 65-93.

[9] Yu. G. Kondrat'ev and Yu. S. Samoylenko, The spaces of trial and generalized functions of infinite number of variables, Rep. Math. Phys., 14 (1978), 325-350.

[10] I. Kubo and S. Takenaka, Calculus on Gaussian white noise I, Proc. Japan Acad., 56A (1980), 376-380 ; II, ibid., 56A (1980), 411-416.

[11] I. Kubo and Y. Yokoi, A remark on the space of testing random variables in the white noise calculus, Nagoya Math. J., 115 (1989), 139-149.

[12] Y.-J. Lee, Analytic version of test functionals, Fourier transform and a characterization of measures in white noise calculus, J. Funct. Anal., 100 (1991), 359-380.

[13] N. Obata, Rotation-invariant operators on white noise functionals, Math. Z., 210 (1992), 69-89.

[14] N. Obata, Fock expansion of operators on white noise functionals, in "Proc. 3rd International Conference on Stochastic Processes, Physics and Geometry (S. Albeverio et al. Eds.)," to appear.

[15] N. Obata, An analytic characterization of symbols of operators on white noise functionals, J. Math. Soc. Japan, to appear.

[16] H. H. Schaefer, "Topological Vector Spaces," 4th corrected printing, SpringerVerlag, 1980.

[17] H. Sugita, Positive generalized Wiener functionals and potential theory over abstract Wiener spaces, Osaka J. Math., 25 (1988), 665-696.

[18] Y. Yamasaki, "Measures on Infinite Dimensional Spaces," Part A, World Scientific, 1985. 
[19] Y. Yokoi, Positive generalized white noise functionals, Hiroshima Math. J., 20 (1990), 137-157.

Department of Mathematics

School of Science

Nagoya University

Nagoya, 464-01 Japan 